\title{
Scaled-Energy Based Spectrum Sensing for Multiple Antennas Cognitive Radio
}

\author{
Michael Dejene Azage ${ }^{1}$, Chaewoo Lee ${ }^{2}$ \\ ${ }^{1,2}$ Department of Electrical and Computer Engineering, Ajou University \\ 206 World cup-ro, Yeongtong-gu, Suwon, Gyeonggi-do 443-749, Republic of Korea \\ [E-mail: ${ }^{1}$ mickyantu@gmail.com, ${ }^{2}$ cwlee@ajou.ac.kr] \\ *Corresponding author : Chaewoo Lee
}

Received August 27, 2017; revised May 10, 2018; accepted May 20, 2018;

published November 30, 2018

\begin{abstract}
In this paper, for a spectrum sensing purpose, we heuristically established a test statistic (TS) from a sample covariance matrix (SCM) for multiple antennas based cognitive radio. The TS is formulated as a scaled-energy which is calculated as a sum of scaled diagonal entries of a SCM; each of the diagonal entries of a SCM scaled by corresponding row's Euclidean norm. On the top of that, by combining theoretical results together with simulation observations, we have approximated a decision threshold of the TS which does not need prior knowledge of noise power and primary user signal. Furthermore, simulation results - which are obtained in a fading environment and in a spatially correlating channel model - show that the proposed method stands effect of noise power mismatch (non-uniform noise power) and has significant performance improvement compared with state-of-the-art test statistics.
\end{abstract}

Keywords: Cognitive radio, noise power mismatch, sample covariance matrix, scaled energy, spectrum sensing. 


\section{Introduction}

Cognitive radio [1] is a promising technology that will improve network utilization by opportunistically accessing licensed primary users signals [2]. It does this by looking for temporally or spectrally or spatially vacant channels [3]. The method for detecting whether a particular channel is occupied by primary user signals or not is called spectrum sensing (SS) [4].

Through SS, either cognitive radios avoid interference to primary users' (PU) signals or use a vacant channel for communication purpose. Hence, SS needs high probability of detection (to avoid interference to PU signals) and low probability of false alarm (for cognitive radio communication). This is the target of SS algorithms. To attain the mentioned target, collaborative or cooperative spectrum sensing (CSS) is devised [5].

There are several CSS mechanisms [6]. For example: (1) Fusion center based [7] (2) Ad-hoc network based [8] (3) Multiple antennas based [9]. The fusion center and ad-hoc network oriented CSS have drawbacks of: (i) Susceptibility to imperfect reporting channel [13] (ii) Requiring common control channel. To overcome these drawbacks, works at [10]-[14] utilized the third scheme: multiple antennas based CSS. In addition, the usage of multiple antennas can enable to devise test statistics that are immune to noise power (variance) uncertainty problem [12][17].

Research works [14][15] show that performance of a cognitive radio is also affected by noise power nature. Here, by nature we mean: whether noise power at output observation of antennas are the same (uniform noise power, UNP) or different (non-uniform noise power, NNP). Report at [14] indicates that performances of different detection algorithms behave differently under the two cases. However, there are algorithms [14][15] which do not show performance fluctuation depending on whether noise power is UNP or NNP.

Motivated by the advantages of using multiple antennas cognitive radio for a SS purpose as discussed in the above two paragraphs, in this manuscript, by relying on a sample covariance matrix (SCM), we heuristically formulated a test statistic (TS). Accordingly, the contributions of this manuscript can be stated as:

i. Without imposing of high computational complexity, we have formulated a detection algorithm for multiple antennas cognitive radio as a sum of scaled-energy which are established by scaling each of the diagonal entries of a SCM by its corresponding row's Euclidean norm. And the performance of the TS formulated is superior compared with state-of-the-art detectors. On the top of that, since the method only considers a sum of scaled diagonal entries, both theoretical and simulation results indicate that it has less computational time.

ii. Also, the performance of the proposed method is noise power nature (UNP or NNP) invariant. In addition to simulation-wise verification, we have theoretically shown this under the signal-free state using an asymptotic analysis.

iii. Moreover, by combining theoretical results with simulation observations, we have approximated a decision threshold of the proposed method. And the decision threshold does not need knowledge of noise power.

Finally, the rest of the paper is organized as follows: section-2 briefly gives related works; section-3 formulates the problem of spectrum sensing using multiple antennas cognitive radio 
with their underlying basic assumptions; section-4 discusses the proposed method; section-5 provides simulation results and analysis; and section- 6 concludes the paper.

\section{Related Works}

The works at [10]-[16] established test statistics by relying on a SCM. This is because of the fact that the SCM contains correlation information when the primary user signals present.

The correlation of a SCM leads to spreading of eigenspectrum when primary user signals exist. Using this advantage, [10]-[12] proposed eigenvalues dependent test statistics. The works at [10] utilized a ratio of the minimum to the maximum eigenvalues. Furthermore, it is assumed to operate in an environment with a single primary user source (rank-1 signal); when numbers of primary user sources are greater than one (rank-P signals [14]), its performance degrades significantly. The works of [11][12] can alleviate the problem of dependency of [10] on a rank of signals. Specifically, [11] (Eigenvalue-to-Moment Ratio, EMR ) used a ratio of the Frobenius norm of a SCM to a square of a trace of a SCM as a particular case. And the work at [13] is based on a Separating Function Estimation Test (SFET) framework to establish test statistics from higher order moments of eigenvalues. Despite the fact that the performances of [10]-[12] are immune to noise variance uncertainty problem [17] when it is the UNP case, their performance decline in the NNP case and we will verify this for [11][12] under the section-5 (results and analysis); for [10], it is already reported in [13].

On the other hand, the authors at [13]-[16] applied non-eigenvalues approach. Their test statistics are directly derived from a SCM. Particularly, [13] (Volume Detector, VD) uses the determinant of a SCM by first dividing each entry of a SCM with their corresponding row's Euclidean norm. Whereas, [14] (Hadamard Ratio Test) uses a ratio of the determinant of a SCM to a product of diagonal entries of a SCM. As reported in [13], the methods [13][14] are robust detection algorithms: their performances remain the same irrespective of whether noise power is UNP or NNP. However, as we will verify through simulation, since both [13] and [14] require computation of determinant, their TS impose high computational time when numbers of antennas get large. Also, they do not have theoretical decision thresholds which make their decision thresholds dependent on a Monte Carlo method. On the other hand, the work at [15] and [16] employee a weighted sum of a sample correlation matrix and the maximum eigenvalue after Cholesky Factorization, respectively. In contrast to [13][14], [15][16] need prior knowledge of noise power that make them impractical.

\section{System Model and Basic Assumptions}

In this paper, bold uppercase, and bold lowercase letters denote matrices and row vectors, respectively, while lightface letters denote scalars. Moreover, $C^{a \times b}$ stands for the complex number with dimension of $a \times b$, where $a$ and $b$ are real numbers; $(.)^{H}$ stands for the complex conjugate transpose; E[.] stands for the expectation operator; $\mathrm{V}[$.$] stands for the$ variance operator; $\operatorname{tr}\left(\right.$.) stands for the trace of a matrix; \|\|$_{F}^{2}$ stands for square of the Frobenius norm of a matrix.

\subsection{Signal Representation}

As depicted in Fig. 1, assume that a cognitive radio (CR) with a number of antennas $M \geq 2$ is employed for sensing signals originating from $L$ independent primary user sources; 
where each of the transmitters is a single antenna based [13]. We further assume that $N$ samples of signals are gathered at each antenna's output observation. Output observation of signals received can be represented as

$$
\left\{\begin{array}{l}
H_{1}: \boldsymbol{X}=\boldsymbol{H} \boldsymbol{S}+\boldsymbol{W} \\
H_{0}: \boldsymbol{X}=\boldsymbol{W}
\end{array}\right.
$$

where $H_{1}$ and $H_{0}$ stand for primary user (PU) signals presence and absence hypotheses, respectively; $\boldsymbol{X} \in C^{M \times N}$ represents the following compact form

$$
\boldsymbol{X}=\left[\begin{array}{lllll}
\boldsymbol{x}_{1} & \boldsymbol{x}_{2} & \boldsymbol{x}_{3} & \cdots & \boldsymbol{x}_{M}
\end{array}\right]^{H}
$$

so that $\boldsymbol{x}_{1}, \boldsymbol{x}_{2}, \boldsymbol{x}_{3}, \cdots, \boldsymbol{x}_{M} \in C^{N \times 1}$ are column vectors containing output observation of signals at each antenna; $\boldsymbol{S} \in C^{L \times N}$ is the compact form representation of PU signals as

$$
\boldsymbol{S}=\left[\begin{array}{lllll}
s_{1} & s_{2} & s_{3} & \cdots & s_{L}
\end{array}\right]^{H},
$$

where $\boldsymbol{s}_{1}, \boldsymbol{s}_{2}, \boldsymbol{s}_{3}, \cdots, \boldsymbol{s}_{L} \in C^{N \times 1}$ are column vectors containing signals originating from each of the PU sources; and $W \in C^{M \times N}$ is the compact matrix form representation for the additive white Gaussian noise (AWGN) as

$$
\boldsymbol{W}=\left[\begin{array}{lllll}
\boldsymbol{w}_{1} & \boldsymbol{w}_{2} & \boldsymbol{w}_{3} & \cdots & \boldsymbol{w}_{M}
\end{array}\right]^{H},
$$

where $\boldsymbol{w}_{1}, \boldsymbol{w}_{2}, \boldsymbol{w}_{3}, \cdots, \boldsymbol{w}_{M} \in C^{N \times 1}$ are column vectors containing AWGN at each output observation of antennas with an assumption that all the entries of $\boldsymbol{w}_{m}$, for all $m=1,2, \cdots, M$, are from the zero-mean circularly symmetric complex normal distribution $\operatorname{CN}\left(0, \sigma_{m}^{2}\right)$; where $\sigma_{m}^{2}$ is the unknown noise variance (power). And, the channel state matrix $\boldsymbol{H} \in C^{M \times L}$, refer to the Fig. 1, has the form

$$
\boldsymbol{H}=\left[\begin{array}{cccc}
h_{11} & h_{12} & \cdots & h_{1 L} \\
h_{21} & h_{22} & \cdots & h_{2 L} \\
\vdots & \vdots & \cdots & \vdots \\
h_{M 1} & h_{M 2} & \cdots & h_{M L}
\end{array}\right]_{M \times L}
$$

where each of the columns of (1d) are from the complex normal distribution with zero-mean and covariance matrix $\Sigma$ [18] such that the entries of $\Sigma$ are given as

$$
\Sigma_{k m}=\frac{J_{0}\left(\sqrt{K^{2}-4 \pi^{2} d_{k m}^{2}+j 4 \pi K d_{k m} \sin \mu}\right)}{J_{0}(K)},
$$

for all $k, m=1,2, \cdots, M ; \quad J_{0}($.$) is the zero-order modified Bessel function; j$ stands for the 

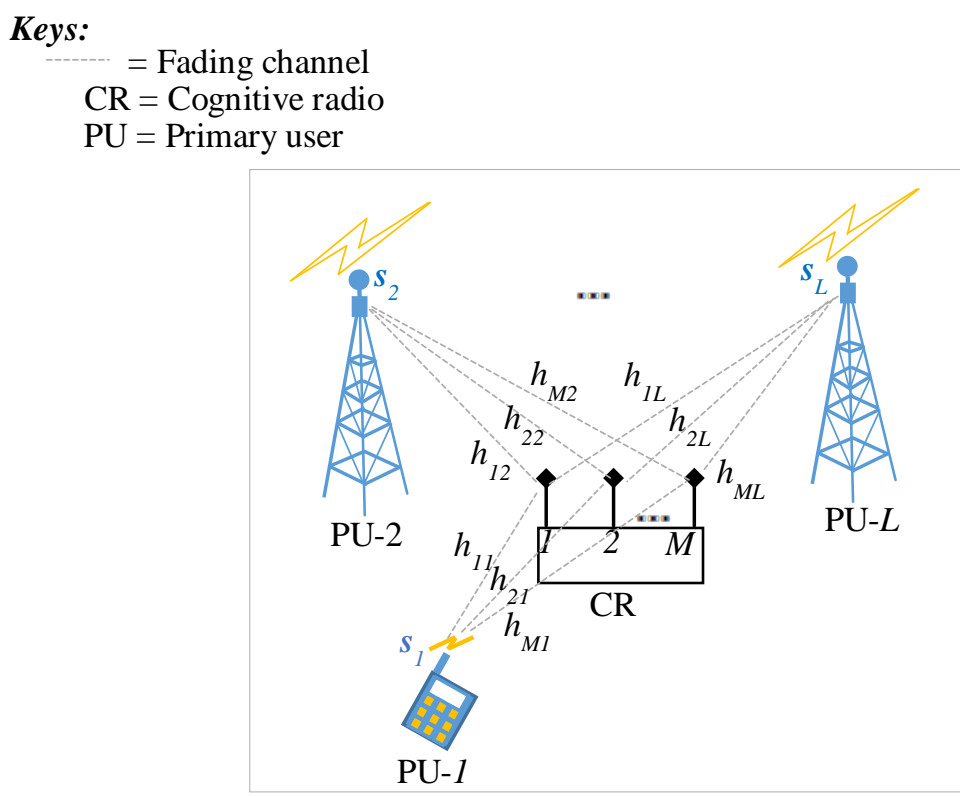

Fig. 1. System diagram: the $h_{11}, h_{21}, \ldots, h_{M L}$ denote the fading channel between each antenna \& PU source, and the $\mathbf{s}_{1}, \mathbf{s}_{2}, \ldots, \mathbf{s}_{\mathrm{L}}$ denote signals from each of the PU sources.

imaginary number $\sqrt{-1} ; K$ controls width of angle of arrival (AoA) which ranges from isotropic scattering when $K=0$ to extremely non-isotropic scattering when $K=\infty$; $\mu \in[-\pi, \pi]$ represents mean direction of AoA; $d_{k m}$ stands for normalized distance between the $k^{\text {th }}$ and the $m^{\text {th }}$ antennas. Modeling $\boldsymbol{H}$ in this manner is called space-time channel correlation model [18] and it resembles a real system as mentioned in [13].

Using (1a)-(1e), each of the column vectors $\boldsymbol{x}_{m} \in C^{N \times 1}$ that makes the compact form $\boldsymbol{X}$, which is given at (1a), is then:

$$
\boldsymbol{x}_{m}=\left(\sum_{k=1}^{L} h_{m k} \boldsymbol{s}_{k}\right)+\boldsymbol{w}_{m},
$$

for all $m=1,2,3, \cdots, M$.

\subsection{Problem Formulation}

Assuming that the signal representation at (1) has both the in-phase and the quadrature output observations, we have adopted a tactic used in [13] as

$$
\boldsymbol{Y}=\left[\begin{array}{lllll}
y_{1} & y_{2} & y_{3} & \cdots & y_{M}
\end{array}\right]^{H}
$$

so that $\boldsymbol{Y} \in C^{M \times 2 N}$ and $\boldsymbol{y}_{1}, \boldsymbol{y}_{2}, \boldsymbol{y}_{3}, \cdots, \boldsymbol{y}_{M} \in C^{2 N \times 1}$ are column vectors. For each of the $\boldsymbol{y}_{1}, \boldsymbol{y}_{2}, \boldsymbol{y}_{3}, \cdots, \boldsymbol{y}_{M}$, the first $N$ samples are from the in-phase and the next $N$ samples are 
from the quadrature output observations of the corresponding $\boldsymbol{x}_{1}, \boldsymbol{x}_{2}, \boldsymbol{x}_{3}, \cdots, \boldsymbol{x}_{M}$ as given at (1f).

By using definition, a sample covariance matrix of $\boldsymbol{Y}$ can be given as

$$
\boldsymbol{R}=\frac{1}{2 N} \boldsymbol{Y} \mathbf{Y}^{H},
$$

where $\boldsymbol{R} \in C^{M \times M}$ and each of its entry is

$$
r_{k m}=\frac{1}{2 N} \sum_{n=1}^{2 N} \boldsymbol{y}_{k}(n) \boldsymbol{y}_{m}^{H}(n),
$$

for all $k, m=1,2, \cdots, M$. The diagonal entries of $\boldsymbol{R}$ provide average energy information, while the off-diagonal entries give similarities information. Furthermore, when a number of samples of a signal are very large, the average magnitude of each entry of $\boldsymbol{R}$ is larger under the state $H_{1}$ than under the state $H_{0}$. Statistically, these can be stated as: $\left|\mathrm{E}\left[r_{k m} \mid H_{1}\right]\right|>$ $\left|\mathrm{E}\left[r_{k m} \mid H_{0}\right]\right|$. This is due to the fact that the output observations of received signals correlate under the state $H_{1}$, whereas they do not correlate when the state is $H_{0}$. And, in this paper, with utilizing this property of $\boldsymbol{R}$, we will consider the problem of spectrum sensing for multiple antennas based cognitive radio by relying on $\boldsymbol{R}$. In addition, we will see the problem of noise power non-uniformity or uncalibrated receivers effect (see paragraph-4 of the section-1).

\section{The Proposed Method: Scaled-Energy Based Spectrum Sensing}

\subsection{Test Statistic from Scaled-Energy}

In this subsection, we heuristically formulate a test statistic from the SCM. We form it as a sum of scaled-energy. By adopting a method used in [13], each of the entries of $\boldsymbol{R}$, which is given at (4), can be scaled as

$$
\hat{r}_{k m}=\frac{r_{k m}}{\tau_{k}}
$$

where

$$
\tau_{k}=\left(\sum_{t=1}^{M} r_{k t} r_{k t}^{H}\right)^{\frac{1}{2}}
$$

is the corresponding row's Euclidean norm for all $k, m=1,2, \cdots, M$. Using (5)(6), let us define $\delta_{k}$ as a scaled energy of the output observed signal at the $k^{\text {th }}$ antenna as 


$$
\delta_{k}=\hat{r}_{k k}=\frac{r_{k k}}{\tau_{k}},
$$

where $k=1,2, \cdots, M$, and $r_{k k}$ is the energy of the output observed signal at $k^{\text {th }}$ antenna and it can be evaluated using (4); and $\tau_{k}$ is as per given at (6). Due to the uncorrelation of the off-diagonal entries under the state $H_{0}$, when there is a large number of samples $N$, the value of $\delta_{k} \mid H_{0}$ approaches to one (we will show this later under the subsection 4.2). In contrast, when the PU signals present or under the state $H_{1}$, since the output observed signals at each antenna correlates among themselves, the value of $\delta_{k} \mid H_{1}$ goes to zero; provide that there is a large number of samples and antennas. Consequently, it is easy to implicitly notice that $\mathrm{E}\left[\delta_{k} \mid H_{0}\right]>\mathrm{E}\left[\delta_{k} \mid H_{1}\right]$. This condition serves as a basis to formulate a test statistic that can discriminate between the binary hypotheses $H_{0}$ and $H_{1}$.

Using (7), we define a test statistic - let us denote it with $T_{S E}$ - as a sum of scaled-energy (SE) as

$$
T_{S E}=\sum_{k=1}^{M} \delta_{k}
$$

With the same reasoning used above, for a large sample size, we can infer that $\mathrm{E}\left[T_{S E} \mid H_{0}\right]>$ $\mathrm{E}\left[T_{S E} \mid H_{1}\right]$. Consequently, a decision rule becomes

$$
\left\{\begin{array}{l}
H_{0}: T_{S E} \geq \gamma_{S E} \\
H_{1}: T_{S E}<\gamma_{S E}
\end{array}\right.
$$

where $\gamma_{S E}$ is a decision threshold that can be determined depending on a desired false alarm probability $P_{F}$. We provide an approximate for $\gamma_{S E}$ in the section-5. But, before that we will see asymptotic properties of the test statistic $T_{S E}$ under the state $H_{0}$. And, the summary of the proposed method is given below in Table 1.

Table 1. Summary of the proposed detection algorithm.

\footnotetext{
Step1: As per (2), find the compact form of $\boldsymbol{Y}$.

Step2: Using (3), compute the SCM $\boldsymbol{R}$.

Step3: Using (7), for all $k=1,2, \cdots, M$, scale the diagonal entries of $\boldsymbol{R}$

Step4: $\quad$ Decide in favor of $H_{1}$ if $T_{S E}<\gamma_{S E}$; otherwise, decide as $H_{0}$.
}

\subsection{Asymptotic Properties of the Test Statistic Under $H_{0}$}

We provide the asymptotic properties of the test statistic under the state $H_{0}$ for a reason: a 
decision threshold is usually easy to determine under the state $H_{0}$ [11]. In order to do this, first we will see asymptotic properties of the diagonal and off-diagonal entries of $\boldsymbol{R}$ (see (4)), and then extend these properties to study asymptotic properties of $T_{S E} \mid H_{0}$.

Lemma-1: Under the state $H_{0}$ and a large number of samples $N$ - for all $k, m=1,2, \cdots, M$ and $k \neq m$ - each of the off-diagonal entries of $\boldsymbol{R}$ asymptotically follows a normal distribution as

$$
r_{k m} \mid H_{0} \sim \mathcal{N}\left(0, \frac{\sigma_{k}^{2} \sigma_{m}^{2}}{2 N}\right),
$$

where $\sigma_{k}^{2}$ and $\sigma_{m}^{2}$ are noise variance at the output observations of the $k^{\text {th }}$ and $m^{\text {th }}$ antennas, respectively.

Proof: Using (4) and the central limit theorem together with the assumptions put in the section-3, Lemma-1 can be easily proofed as

$$
\mathrm{E}\left[r_{k m} \mid H_{0}\right]=\frac{1}{2 N} \sum_{n=1}^{2 N} \mathrm{E}\left[\boldsymbol{y}_{k}(n) \mid H_{0}\right] \mathrm{E}\left[\boldsymbol{y}_{m}(n) \mid H_{0}\right]=0
$$

and

$$
\begin{gathered}
\mathrm{V}\left[r_{k m} \mid H_{0}\right]=\sum_{n=1}^{2 N} \frac{1}{4 N^{2}} \mathrm{~V}\left[\left(\boldsymbol{y}_{k}(n) \mid H_{0}\right)\left(\boldsymbol{y}_{m}(n) \mid H_{0}\right)\right] \\
=\frac{1}{4 N^{2}} \sum_{n=1}^{2 N}\left(\mathrm{E}\left[\boldsymbol{y}_{k}^{2}(n) \mid H_{0}\right] \mathrm{E}\left[\boldsymbol{y}_{m}^{2}(n) \mid H_{0}\right]-\left(\mathrm{E}\left[\boldsymbol{y}_{k}(n) \mid H_{0}\right] \mathrm{E}\left[\boldsymbol{y}_{m}(n) \mid H_{0}\right]\right)^{2}\right) \\
=\frac{\sigma_{k}^{2} \sigma_{m}^{2}}{2 N} .
\end{gathered}
$$

Lemma-2: Under the state $H_{0}$ and a large number of samples $N$ - for all $k=1,2, \cdots, M-$ each of the diagonal entries of $\boldsymbol{R}$ asymptotically follows a normal distribution as

$$
r_{k k} \mid H_{0} \sim \mathcal{N} \mathcal{N}\left(\sigma_{k}^{2}, \frac{\sigma_{k}^{4}}{N}\right),
$$

where $\sigma_{k}^{2}$ is noise variance at the output observation of the $k^{\text {th }}$ antenna.

Proof: Using (4) and the central limit theorem together with assumptions given in the section-3, Lemma-2 can be proofed as

$$
\mathrm{E}\left[r_{k k} \mid H_{0}\right]=\frac{1}{2 N} \sum_{n=1}^{2 N} \mathrm{E}\left[y_{k}^{2}(n) \mid H_{0}\right]=\sigma_{k}^{2}
$$


and

$$
\begin{gathered}
\mathrm{V}\left[r_{k k} \mid H_{0}\right]=\sum_{n=1}^{2 N} \frac{1}{4 N^{2}} \mathrm{~V}\left[\boldsymbol{y}_{k}^{2}(n) \mid H_{0}\right] \\
=\frac{1}{4 N^{2}} \sum_{n=1}^{2 N}\left(\mathrm{E}\left[\boldsymbol{y}_{k}^{4}(n) \mid H_{0}\right]-\left(\mathrm{E}\left[\boldsymbol{y}_{k}^{2}(n) \mid H_{0}\right)^{2}\right)\right. \\
=\frac{1}{4 N^{2}} \sum_{n=1}^{2 N}\left(3 \sigma_{k}^{4}-\left(\sigma_{k}^{2}\right)^{2}\right)=\frac{\sigma_{k}^{4}}{N} .
\end{gathered}
$$

From the Lemma-1 and the Lemma-2, we can conclude the followings:

i. $\quad$ under the state $H_{0}$, the entries of $\boldsymbol{R} \mid H_{0}$ are dependent on the noise power,

ii. due to the signals' non-correlation, when $N$ is very large, each of the off-diagonal entries of $\boldsymbol{R} \mid H_{0}$ goes to zero (Lemma-1) and

iii. each of the diagonal entries of $\boldsymbol{R} \mid H_{0}$ goes to noise power (Lemma-2).

However, as subsequently will be shown at Lemma-3, the scaling parameter $\tau$ enables non-dependency of the diagonal values of $\boldsymbol{R} \mid H_{0}$ on the noise power.

Lemma-3: Under the state $H_{0}$ and a large number of samples $N$, regardless of the noise power, the expected value of the scaled energy $\delta_{k} \mid H_{0}$ at the output observation of the $k^{\text {th }}$ antenna, which is given at (7), asymptotically approaches one from the left as

$$
\mathrm{E}\left[\delta_{k} \mid H_{0}\right] \rightarrow 1
$$

Proof: Lemma-3 can be proofed using the results at the Lemma-1 and Lemma-2. The scaling parameter $\tau_{k}$, which is given at (6), for a large number of samples can be approximated as

$$
\mathrm{E}\left[\tau_{k} \mid H_{0}\right]=\left[\left(r_{k k}^{2}+\sum_{m=1, m \neq k}^{M} r_{k m} r_{k m}^{H}\right)^{2}\right] .
$$

From the Lemma-1, since each of the off-diagonal entries of $\boldsymbol{R} \mid H_{0}$ goes to zero for a large number of samples, the following can be approximated at (12a):

$$
\sum_{m=1, m \neq k}^{M} r_{k m} r_{k m}^{H} \approx 0
$$

so that

$$
\mathrm{E}\left[\tau_{k} \mid H_{0}\right] \approx \mathrm{E}\left[\left(r_{k k}^{2} \mid H_{0}\right)^{\frac{1}{2}}\right]=\mathrm{E}\left[r_{k k} \mid H_{0}\right] .
$$

With further using Lemma-2, (12c) above is 


$$
\mathrm{E}\left[\tau_{k} \mid H_{0}\right] \approx \sigma_{k}^{2},
$$

where $\sigma_{k}^{2}$ is the noise power at the output observation of the $k^{\text {th }}$ antenna for all $k=1,2, \ldots, M$. Using (12d), under a large number of samples and the state $H_{0}$, the scaled energy at (7) can be approximated as

$$
\delta_{k} \mid H_{0} \approx \frac{r_{k k} \mid H_{0}}{\mathrm{E}\left[\tau \mid H_{0}\right]}
$$

so that using Lemma-2

$$
\mathrm{E}\left[\delta_{k} \mid H_{0}\right] \rightarrow \frac{1}{\sigma_{k}^{2}} \mathrm{E}\left[r_{k k} \mid H_{0}\right]=1 .
$$

From the Lemma-3 above, under the state $H_{0}$ and large number of samples, we can infer that the expected value of the scaled-energy $\delta_{k} \mid H_{0}$ is independent of the noise power.

Lemma-4: Under the state $H_{0}$ and a large number of samples, regardless of the noise power, the expected value of the test statistic $T_{S E} \mid H_{0}$, which is given at (8), asymptotically approaches $M$ from the left as

$$
\mathrm{E}\left[T_{S E} \mid H_{0}\right] \rightarrow M
$$

where $M$ is the number of antennas of the cognitive radio.

Proof: By using the Lemma-3, Lemma-4 can be easily proofed as

$$
\mathrm{E}\left[T_{S E} \mid H_{0}\right]=\sum_{k=1}^{M} \mathrm{E}\left[\delta_{k} \mid H_{0}\right] \rightarrow \sum_{k=1}^{M} 1=M .
$$

Lemma-4 tells us that the asymptotic expected value of $T_{S E} \mid H_{0}$ under a large number of samples is independent of the noise power. Together with simulation observations, in the section-5 (subsection 5.1), we will utilize Lemma-4 in approximating the decision threshold $\gamma_{S E}$ which is given at (9).

\subsection{Computational Complexity}

The computational complexities for the proposed method, EMR, SFET 1 and VD are given in Table 2. Since all the detectors are based on a SCM, they require $\mathrm{O}\left(M^{2} N\right)$ [12] for a SCM computation. Particularly, in our proposed work (SE), there is about $\mathrm{O}\left(M^{2}\right)$ for computation of the Euclidean norm, division of each entry of a row by its corresponding Euclidean norm and summing all the scaled-energy values to get the final test statistic $T_{S E}$. As a result, the 
overall complexity of the proposed method is $\mathrm{O}\left(M^{2} N\right)+\mathrm{O}\left(M^{2}\right)$. In [13], the computational complexity is not provided for the VD test statistic; however, since it involves the computation of a determinant, its complexity is supposed to be inferior to the proposed method.

Table 2. Complexities comparison using the Big-O.

\begin{tabular}{lc}
\hline Detectors & Complexities \\
\hline \hline SE (Proposed): & $\mathrm{O}\left(M^{2} N\right)+\mathrm{O}\left(M^{2}\right)$ \\
EMR [11]: & $\mathrm{O}\left(M^{2} N\right)+\mathrm{O}\left(M^{2}\right)$ \\
SFET $_{1}[12]:$ & $\mathrm{O}\left(M^{2} N\right)+\mathrm{O}\left(M^{2.373}\right)$ \\
VD [13]: & --- \\
\hline
\end{tabular}

\section{Results and Analysis}

We have used the simulation environment of [13] which assumes that all the $L$ primary user transmitters independently transmit QPSK signals, symbols $\left( \pm \frac{1}{\sqrt{2}}, \pm \frac{1}{\sqrt{2}}\right)$, with equal power and probabilities. And the signals are subjected to the fading environment represented in the section-2 at (1) with the space-time channel correlation model: the channel state $\boldsymbol{H}$. The parameters that describe $\boldsymbol{H}$ are set as follows: the angle of arrival (AoA) $\mu=\frac{\pi}{2}$; the width of AoA $K=80$; the normalized distance between adjacent antennas $d_{k m}=0.5$, provide that all the antennas are arranged in a linear array fashion and mutually not coupled. In addition, each column of $\boldsymbol{H}$ is normalized so that signal-to-noise ratio (SNR) is defined as $\operatorname{SNR}=\frac{\operatorname{tr}\left(\boldsymbol{R}_{s}\right) / L}{\operatorname{tr}\left(\boldsymbol{R}_{w}\right) / M}$, where $\left.\boldsymbol{R}_{S}=\mathrm{E} \mid \boldsymbol{S} \boldsymbol{S}^{H}\right]$ and $\left.\boldsymbol{R}_{w}=\mathrm{E} \mid \boldsymbol{W} \boldsymbol{W}^{H}\right][13]$.

For the purpose of comparing with our work Scaled-Energy (SE), we selected state-of-the-art detectors: Volume Detector (VD [13]), Eigenvalue-Moment-Ratio (EMR [11]), and Separating Function Estimation Test (SFET ${ }_{1}$ [12]). It is important to notice that, in [12], they proposed two test statistics: $\mathrm{SFET}_{1}$ and $\mathrm{SFET}_{2}$. Since the performance of $\mathrm{SFET}_{1}$ is slightly superior to $\mathrm{SFET}_{2}$, we selected $\mathrm{SFET}_{1}$.

Unless otherwise mentioned, performances are considered over 50,000 independent Monte Carlo trials. And during each trial, the value of $\boldsymbol{H}$ is fixed constant. Furthermore, the evaluation is considered in the two cases: (1) Uniform noise power (UNP) and (2) Non-uniform noise power (NNP); (see paragraph-4 of the section-1).

\subsection{Decision Threshold}

\subsubsection{Approximation}

In this subsection, under the state $H_{0}$, we approximate the decision threshold of the proposed method using simulation observations together with the Lemma-4. To do this, first additive white Gaussian noise, see (1c), is generated at each of the antenna's inphase and 
quadrature output observations. Using the generated noise, for a total number of independent trials of $5 \times 10^{4}$, we have computed $T_{S E} \mid H_{0}$. The obtained value of $T_{S E} \mid H_{0}$ at each trial is recorded in order to plot its histogram as displayed in Fig. 2, Fig. 3 and Fig. 4. As it can be clearly seen when number antennas and number of samples increase, the shape of the histograms approximate the normal distribution. And it is important to notice that the 'Frequency' label along the y-axis of the histograms refers to the number of counts or observations.
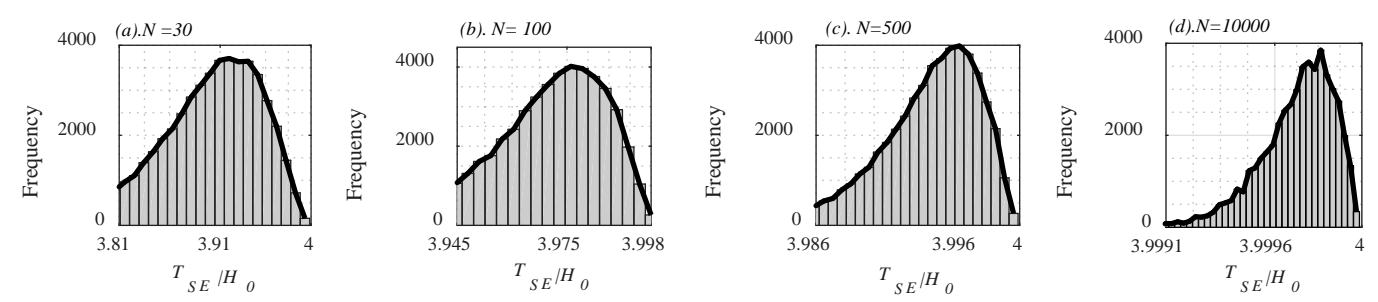

Fig. 2. Histograms obtained by making the number of bins 50 and number of trials 50,000 for $M=4$.
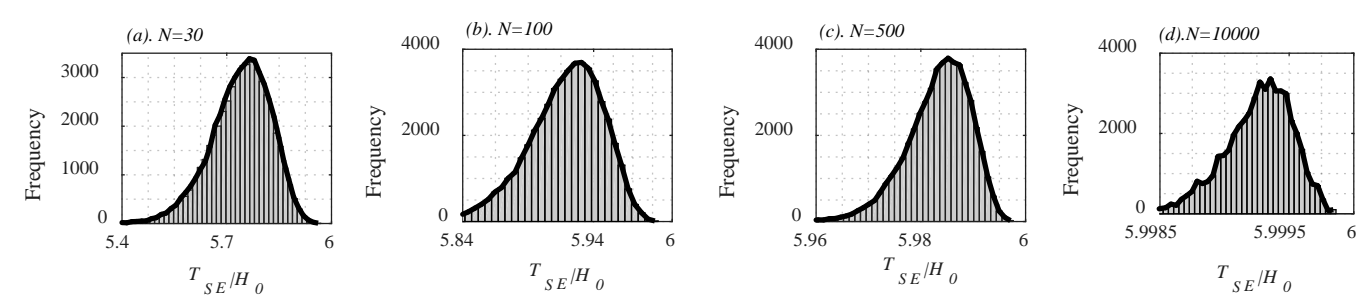

Fig. 3. Histograms obtained by making the number of bins 50 and number of trials 50,000 for $M=6$.
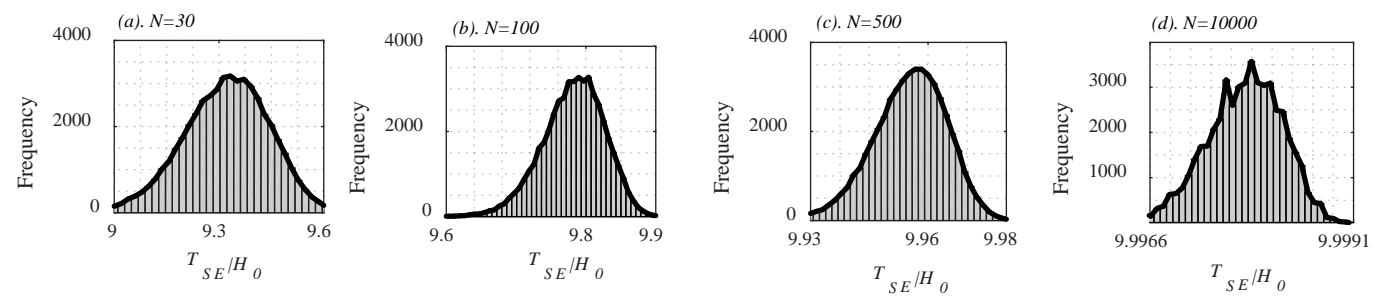

Fig. 4. Histograms obtained by making the number of bins 50 and number of trials 50,000 for $M=10$.

Lemma-4 tells us that the expected value of the test statistic $T_{S E} \mid H_{0}$ asymptotically approaches $M$ from the left. Based on the Lemma-4, let us write the simulation obtained sample mean value of $T_{S E} \mid H_{0}$ as

$$
\mu_{\text {sim }} \mid H_{0}=M-\varepsilon,
$$

for some very small random positive real number $\varepsilon$ that tends to zero as the number of sample increases. From (14), the value of $\varepsilon$ can be evaluated as $\varepsilon=M-\mu_{\text {sim }} \mid H_{0}$. The values of $\mu_{\text {sim }} \mid H_{0}$ - which are evaluated from the data used to plot the histograms in Fig. 2, Fig. 3 and Fig. 4 -are given in Table 3. As it is clear to see from the table, the value of $\varepsilon$ tends to zero as the number of sample increases. This simulation observation strongly supports Lemma-4 
which states that under a large number of samples, the expected value of $T_{S E} \mid H_{0}$ asymptotically tends to $M$. With this, for practical purposes, we can approximate the simulation obtained mean value of the $T_{S E} \mid H_{0}$ as

$$
\mu_{\text {sim }} \mid H_{0} \approx M \text {. }
$$

Also, we have evaluated simulation sample variance $\sigma_{\text {sim }}^{2} \mid H_{0}$ of the $T_{S E} \mid H_{0}$ and displayed its value in Table 3. Using the simulation results obtained, we have found that the sample variance $\sigma_{\text {sim }}^{2} \mid H_{0}$ can be approximated as

$$
\sigma_{\text {sim }}^{2} \mid H_{0}=\frac{M^{2}}{N^{2}}
$$

and for comparison purposes, the value of $\sigma_{\text {sim }}^{2} \mid H_{0}$ is provided along with its approximate $\frac{M^{2}}{N^{2}}$ in Table 3 .

\begin{tabular}{|c|c|c|c|c|c|}
\hline \multicolumn{2}{|c|}{$\begin{array}{l}\text { Histograms together with the }(M, N) \\
\text { considered }\end{array}$} & $\mu_{\text {sim }} \mid H_{0}$ & $\varepsilon=M-\mu_{\text {sim }} \mid H_{0}$ & $\sigma_{\text {sim }}^{2} \mid H_{0}$ & $\frac{M^{2}}{N^{2}}$ \\
\hline \multirow{4}{*}{$\begin{array}{l}\text { Histogram-1 } \\
\quad \text { (Fig. 2) }\end{array}$} & $(M, N)=(4,30)$ & 3.9201 & 0.0799 & 0.0120 & $\begin{array}{l}0.0178 \\
\end{array}$ \\
\hline & $(M, N)=(4,100)$ & 3.9763 & 0.0237 & 0.0011 & 0.0016 \\
\hline & $(M, N)=(4,500)$ & 3.9972 & 0.0028 & $6.1 \times 10^{-5}$ & $6.40 \times 10^{-5}$ \\
\hline & $(M, N)=\left(4,10^{4}\right)$ & 3.9997 & 0.0003 & $1.43 \times 10^{-7}$ & $1.60 \times 10^{-7}$ \\
\hline \multirow{4}{*}{$\begin{array}{l}\text { Histogram-2 } \\
\quad \text { (Fig. 3) }\end{array}$} & $(M, N)=(6,30)$ & 5.7104 & 0.2896 & 0.0330 & 0.0400 \\
\hline & $(M, N)=(6,100)$ & 5.9402 & 0.0598 & 0.0029 & 0.0036 \\
\hline & $(M, N)=(6,500)$ & 5.9811 & 0.0189 & $1.17 \times 10^{-4}$ & $1.44 \times 10^{-4}$ \\
\hline & $(M, N)=\left(6,10^{4}\right)$ & 5.9993 & 0.0007 & $3.26 \times 10^{-7}$ & $3.60 \times 10^{-7}$ \\
\hline \multirow{4}{*}{$\begin{array}{l}\text { Histogram-3 } \\
\quad \text { (Fig. 4) }\end{array}$} & $(M, N)=(10,30)$ & 9.2506 & 0.7494 & 0.1063 & 0.1111 \\
\hline & $(M, N)=(10,100)$ & 9.7850 & 0.2150 & 0.0084 & 0.0100 \\
\hline & $(M, N)=(10,500)$ & 9.9583 & 0.0417 & $3.38 \times 10^{-4}$ & $4.00 \times 10^{-4}$ \\
\hline & $(M, N)=\left(10,10^{4}\right)$ & 9.9977 & 0.0023 & $0.75 \times 10^{-6}$ & $1.00 \times 10^{-6}$ \\
\hline
\end{tabular}

Table 3. Statistical values of the histograms obtained from simulation.

Using the results at (15)(16), and the shape of the histograms which are approximately bell-shaped together with the decision rule given at (9), the decision threshold $\gamma_{S E}$ of the proposed method at a desired false alarm probability of $P_{F}$ is approximated as

$$
\gamma_{S E} \approx M-\frac{M}{N} Q^{-1}\left(1-P_{F}\right)
$$


where $Q^{-1}$ is an inverse of the $Q$-function.

\subsubsection{Relative Error of a Decision Threshold}

The accuracy of theoretically calculated decision thresholds can be evaluated using a metric called Relative Error (RE) which is given in [13] as

$$
R E=\frac{\left|\gamma_{\text {sim }}-\gamma_{\text {theo }}\right|}{\gamma_{\text {sim }}} \times 100
$$

where $\gamma_{s i m}$ is simulation obtained decision threshold which can be obtained by relying on a Monte-Carlo method, and $\gamma_{\text {theo }}$ is a theoretically approximated decision threshold (in our case, $\gamma_{S E}$ at (17) replaces $\gamma_{\text {theo }}$ ).

The values of $R E$ for some selected number of antennas and samples are provided in Table 4. Compared with the EMR and $\mathrm{SFET}_{1}$, the proposed method (SE) has the smallest $R E$, whereas $\mathrm{SFET}_{1}$ has the largest $R E$. The $R E$ for the VD is not included since it has no theoretical decision threshold.

Remark-1: The Monte-Carlo method based decision thresholds of the Table 4 are obtained under the case uniform noise power (UNP) of $O d B$. We have not given $R E$ values under the case non-uniform noise power (NNP). Because, under this case, the performances of EMR and $\mathrm{SFET}_{1}$ are noise power dependent as we will show this under the subsection 5.2.

Table 4. RE in a percentage of the proposed method (SE), EMR and $\mathrm{SFET}_{1}$.

\begin{tabular}{|c|c|c|c|c|c|c|c|}
\hline \multirow{2}{*}{\multicolumn{2}{|c|}{$\begin{array}{l}\text { Detectors together } \\
\text { with the }(M, N) \\
\text { considered }\end{array}$}} & \multicolumn{3}{|c|}{ When $P_{F}=10^{-3}$ : } & \multicolumn{3}{|c|}{ When $P_{F}=10^{-2}$ : } \\
\hline & & \multirow{2}{*}{ 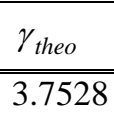 } & \multirow{2}{*}{$\begin{array}{l}\gamma_{\text {sim }} \\
3.8031\end{array}$} & \multirow{2}{*}{$\begin{array}{l}R E(\%) \\
1.32\end{array}$} & \multirow{2}{*}{$\begin{array}{l}\gamma_{\text {theo }} \\
3.8139\end{array}$} & \multirow{2}{*}{$\frac{\gamma_{\text {sim }}}{3.8441}$} & \multirow{2}{*}{$\begin{array}{l}R E(\%) \\
\mathbf{0 . 2 0}\end{array}$} \\
\hline$M=4$ & $\mathrm{SE}$ & & & & & & \\
\hline \multirow{2}{*}{$N=50$} & EMR & 1.1674 & 1.1857 & 1.54 & 1.1458 & 1.1503 & 0.39 \\
\hline & $\mathrm{SFET}_{1}$ & 0.0347 & 0.0314 & 10.51 & 0.0308 & 0.0272 & 13.23 \\
\hline \multirow{3}{*}{$\begin{array}{l}M=4, \\
N=100\end{array}$} & $\mathrm{SE}$ & 3.8764 & 3.8889 & 0.32 & 3.9069 & 3.9178 & 0.29 \\
\hline & EMR & 1.0837 & 1.0891 & 0.50 & 1.0729 & 1.0767 & 0.35 \\
\hline & $\mathrm{SFET}_{1}$ & 0.0253 & 0.0235 & 7.66 & 0.0233 & 0.0215 & 8.37 \\
\hline \multirow{3}{*}{$\begin{array}{l}M=6, \\
N=200\end{array}$} & SE & 5.9073 & 5.9072 & $0.17 \times 10^{-4}$ & 5.9302 & 5.9261 & 0.07 \\
\hline & EMR & 1.0519 & 1.0522 & 0.03 & 1.0464 & 1.0475 & 0.11 \\
\hline & $\mathrm{SFET}_{1}$ & 0.0063 & 0.0061 & 3.28 & 0.0060 & 0.0058 & 3.45 \\
\hline
\end{tabular}

\subsection{Decision Threshold Sensitivity Factor}

Under this subsection, we see whether a decision threshold is affected by noise power mismatch or not. But, first, we define a new parameter that describes a sensitivity of a decision threshold as 


$$
\beta=\left|\frac{\gamma_{U N P}-\gamma_{N N P}}{\gamma_{U N P}}\right| \times 100,
$$

where $\gamma_{U N P}$ and $\gamma_{N N P}$ are simulations obtained decision thresholds under the UNP and the $N N P$ cases, respectively. So, $\beta$ describes relative percentage deviation of a decision threshold under the NNP from that obtained under the UNP case. A small value of $\beta$ describes a minimal variation of a decision threshold which is preferable.

The tabular results displayed in Table 5 shows the values of $\gamma_{U N P}, \gamma_{N N P}$ and $\beta$ at desired false alarm probabilities of $P_{F}=10^{-3}$ and $P_{F}=10^{-2}$ by fixing $N=50, M=4$ and $L=1$. Particularly, the $\gamma_{N N P}$ values are obtained when the noise power are $\left[\sigma_{1}^{2}=1, \sigma_{2}^{2}=1.7, \sigma_{3}^{2}=-0.7, \sigma_{4}^{2}=-2\right] d B$. Under all the circumstances, the proposed method (SE) has the lowest $\beta$ value, while the SFET $_{1}$ has the largest $\beta$ value. Unlike the EMR and the $\mathrm{SFET}_{1}$, for the robust detectors (SE and VD), small values of $\beta$ means decision thresholds are less sensitive to noise power mismatch and makes them practical. In the subsequent parts of this subsection, we will see the theoretical reasons for why the performance of the EMR and the $\mathrm{SFET}_{1}$ are noise power dependent under the case NNP and why the performances of the proposed method (SE) and VD are noise power invariant.

Table 5. The $\beta$ values when UNP of $O d B$ and $N N P$ of $[1,1.7,-0.7,-2] d B$ at $N=50$ and $L=1$.

\begin{tabular}{|c|c|c|c|c|c|c|}
\cline { 2 - 7 } & \multicolumn{3}{|c|}{ When $P_{F}=10^{-3}:$} & \multicolumn{3}{c|}{ When $P_{F}=10^{-2}:$} \\
\hline \hline Detectors & $\gamma_{U N P}$ & $\gamma_{N N P}$ & $\beta(\%)$ & $\gamma_{U N P}$ & $\gamma_{N N P}$ & $\beta(\%)$ \\
\hline \hline SE & 3.8031 & 3.7677 & $\mathbf{0 . 9 3}$ & 3.8441 & 3.8213 & $\mathbf{0 . 5 9}$ \\
\hline VD & -0.4322 & -0.4620 & $\mathbf{6 . 8 9}$ & -0.3322 & -0.3601 & $\mathbf{8 . 4 0}$ \\
\hline EMR & 1.1385 & 1.3285 & $\mathbf{1 6 . 6 9}$ & 1.1058 & 1.2702 & $\mathbf{1 3 . 4 4}$ \\
\hline SFET $_{1}$ & 0.0314 & 0.0584 & $\mathbf{8 5 . 9 9}$ & 0.0272 & 0.0480 & $\mathbf{7 6 . 4 7}$ \\
\hline
\end{tabular}

For a large number of samples, due to the non-correlation of signals, the SCM $\boldsymbol{R} \mid H_{0}$ is a diagonal matrix with each diagonal entries approximately representing noise power at each antenna's output observation. This is because as $N \rightarrow \infty$, the SCM converges to a statistical mean value [19] which can be represented as

$$
\lim _{N \rightarrow \infty} R \mid H_{0} \cong \mathrm{E}\left[R \mid H_{0}\right],
$$

and refer to the Lemma-1 and Lemma -2 for an asymptotic distribution of each of the off-diagonal and the diagonal entries, respectively. Next, we will see the asymptotic properties of the test statistics of the EMR, the SFET 1 and the VD under the state $H_{0}$ with an assumption of a large number of samples. As mentioned previously, doing this will enable us to 
understand reasons why the performances of the EMR and the $\mathrm{SFET}_{1}$ are different under UNP and NNP cases while why that of the proposed and VD are unaffected by noise power:

i. The test statistic of the EMR [11] can be re-expressed as

$$
T_{E M R}=\frac{D_{1}}{D_{2}} \underset{H_{0}}{\stackrel{H_{1}}{\geq}} \gamma_{E M R}
$$

where $D_{1}=\frac{1}{M}\|\boldsymbol{R}\|_{F}^{2}, D_{2}=\left(\frac{1}{M} \operatorname{tr}(\boldsymbol{R})\right)^{2}$ and $\gamma_{E M R}$ is a decision threshold for the test statistic $T_{E M R}$. When noise power is the UNP case, $D_{1} \mid H_{0}$ and $D_{2} \mid H_{0}$ tend to

$$
\lim _{N \rightarrow \infty} D_{1} \mid H_{0} \cong \sigma^{4}
$$

and

$$
\lim _{N \rightarrow \infty} D_{2} \mid H_{0} \cong \sigma^{4}
$$

respectively; where $\sigma^{4}$ denotes uniform noise power at each antenna's output observation. As given at (21a) and (21b), for the UNP case, both the numerator (i.e. $D_{1} \mid H_{0}$ ) and the denominator (i.e. $D_{2} \mid H_{0}$ ) asymptotically approach the same value. As a result, regardless of the noise power, their ratio goes to a unity value. In the contrast, when noise power is the NNP case, the $D_{1} \mid H_{0}$ and $D_{2} \mid H_{0}$ tend to

$$
\lim _{N \rightarrow \infty} D_{1} \mid H_{0} \cong \frac{1}{M} \sum_{k=1}^{M} \sigma_{k}^{4}
$$

and

$$
\lim _{N \rightarrow \infty} D_{1} \mid H_{0} \cong\left(\frac{1}{M} \sum_{k=1}^{M} \sigma_{k}^{2}\right)^{2}=\frac{1}{M^{2}}\left(\sum_{k=1}^{M} \sigma_{k}^{4}+\sum_{r=1}^{M} \sum_{q=1}^{M} \sigma_{r}^{2} \sigma_{q}^{2}\right), r \neq q,
$$

respectively; where $\sigma_{k}^{2}$ denotes non-uniform noise power at each antenna's output observations for $k=1,2, \cdots, M$. The results at (21c) and (21d) imply that the ratio of the asymptotic values of $D_{1} \mid H_{0}$ and $D_{2} \mid H_{0}$ does not approach a specifically defined value; it is noise power variant. This indicates that under the UNP and the NNP cases, the asymptotic values of the test statistic $T_{E M R} \mid H_{0}$ are different which indirectly results in different performances under the two cases.

ii. In [12], the test statistic of the $\mathrm{SFET}_{1}$ can be re-expressed as 


$$
T_{\text {SFE } T_{1}}=\frac{D_{3}}{D_{4}} \underset{H_{0}}{\stackrel{H_{1}}{\geq}} \gamma_{\text {SFET }},
$$

where $D_{3}=\operatorname{tr}\left(\boldsymbol{R}^{4}\right), \quad D_{4}=\operatorname{tr}(\boldsymbol{R})^{4}$, and $\gamma_{S F E T_{1}}$ is the decision threshold for test statistic $T_{S F E T_{1}}$. When noise power is the UNP case, the $D_{3} \mid H_{0}$ and $D_{4} \mid H_{0}$ tend to

$$
\lim _{N \rightarrow \infty} D_{3} \mid H_{0} \cong \sigma^{8}
$$

and

$$
\lim _{N \rightarrow \infty} D_{4} \mid H_{0} \cong \sigma^{8}
$$

respectively; where $\sigma^{2}$ denotes uniform noise power at each antenna's output observation. On the other hand, when noise power is the NNP case, the $D_{3} \mid H_{0}$ and $D_{4} \mid H_{0}$ asymptotically go to

$$
\lim _{N \rightarrow \infty} D_{3} \mid H_{0} \cong \sum_{k=1}^{M} \sigma_{k}^{8}
$$

and

$$
\begin{gathered}
\lim _{N \rightarrow \infty} D_{4} \mid H_{0} \cong\left(\sum_{k=1}^{M} \sigma_{k}^{2}\right)^{4} \\
=\sum_{k=1}^{M} \sigma_{k}^{8}+\sum_{r=1}^{M} \sum_{q=1}^{M} \sigma_{r}^{4} \sigma_{q}^{4}+2 \sum_{t=1}^{M} \sum_{r=1}^{M} \sum_{q=1}^{M} \sigma_{t}^{4} \sigma_{r}^{2} \sigma_{q}^{2}+\left(\sum_{r=1}^{M} \sum_{q=1}^{M} \sigma_{r}^{2} \sigma_{q}^{2}\right)^{2}, r \neq q,
\end{gathered}
$$

respectively; where $\sigma_{k}^{2}$ denotes non-uniform noise power at each antenna's output observations for $k=1,2, \cdots, M$. Under the UNP case, the ratio of the asymptotic values of $D_{3} \mid H_{0}$ (22a) and $D_{4} \mid H_{0}$ (22b) goes to a unity value, while under the NNP case, the ratio of (22c) to (22d) is noise power variant. Consequently, the performances of the test statistic $T_{S F E T_{1}}$ are different under the UNP and the NNP cases.

iii. The VD's test statistic is given in [13] as

$$
T_{V D}=\log (\operatorname{det}(\hat{\boldsymbol{R}}))^{\geq} \gamma_{H_{1}}^{H_{0}},
$$

where the entries of $\hat{\boldsymbol{R}}$ are as per (5), and $\gamma_{V D}$ is the decision threshold for the test statistic $T_{V D}$. Regardless of the noise power, the test statistic $T_{V D} \mid H_{0}$ asymptotically goes to 


$$
\lim _{N \rightarrow \infty} T_{V D} \mid H_{0} \cong 0
$$

The result at (29a) is a consequence of the Lemma-3 and using the property that determinant of a diagonal matrix is simply the product of the diagonal entries. This indicates that $T_{V D} \mid H_{0}$ is noise power invariant.

iv. For the proposed method, the asymptotic property is given at the Lemma-4 which shows that regardless of the noise power, asymptotically, the test statistic $T_{S E} \mid H_{0}$ approaches $M$ (i.e. the number of antennas).

\subsection{ROC and Detection Probability}

The figures in Fig. 5 and Fig. 6 illustrate Receiver Operating Characteristics (ROC) for $S N R=-5 d B$. The ROC curves of Fig. 5 is obtained at a relatively large number of samples ( $N=50$ when $L=1$ ), while that of Fig. 6 is obtained at a relatively small number of samples (in a sample starving environment of $N=8$ when $L=5$ ). On the other hands, the figures Fig. 7 and Fig. 8 show detection probabilities at a specific false alarm probability of $P_{F}=10^{-3}$. The (a) sub-figures are obtained in the UNP case while the (b) sub-figures are obtained in the NNP case.

Generally, the EMR and the SFET 1 show performance differences under the UNP and the NNP cases, whereas the proposed method and VD show robustness: their performance do not fluctuate with the noise power mismatch. For example, from the Fig. 6, under the UNP case and $S N R=-5 d B$, both the EMR and $\mathrm{SFET}_{1}$ have detection probability of about 0.88 . However, under the similar circumstance, but the NNP of $[1,1.7,-0.7,-2] d B$, their performances declined to around 0.17 . The theoretical reasons for the dissimilarity of the detection probability under the UNP and the NNP cases can be similarly justified using the reasoning techniques provided under the subsection 5.2.
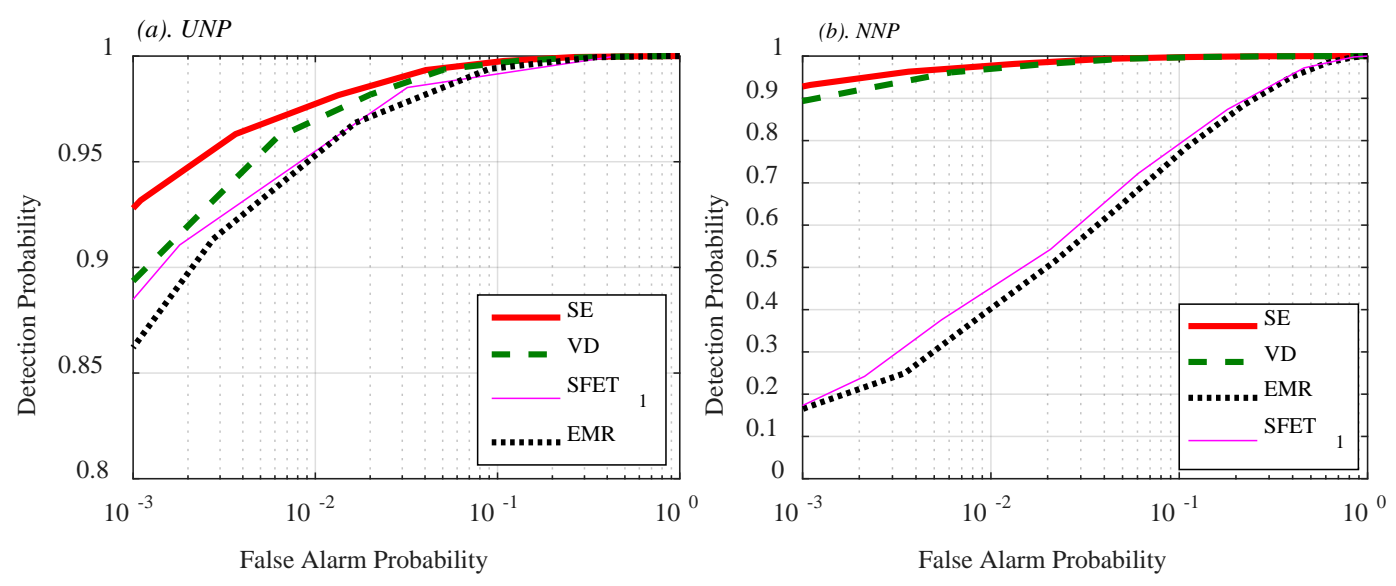

Fig. 5. ROC curves by fixing $L=1, S N R=-5 d B, M=4, N=50$ : (a) UNP of $0 \mathrm{~dB}$ (b) NNP of [1, $1.7,-0.7,-2] d B$. 

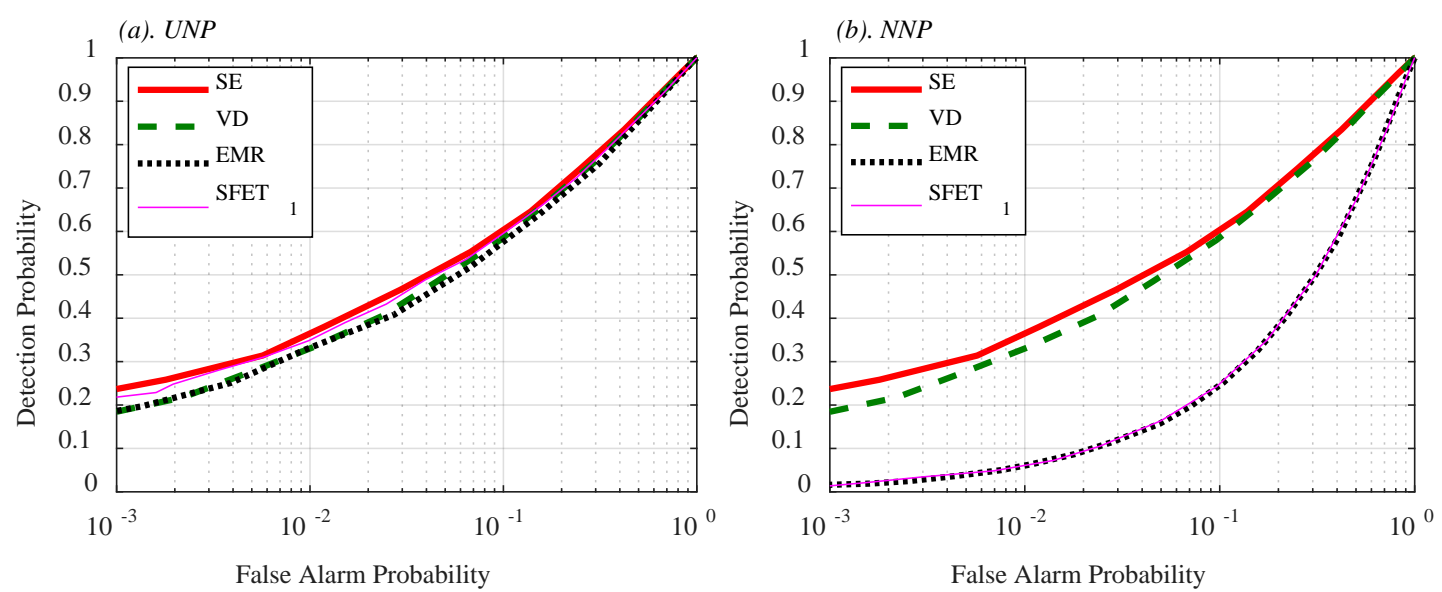

Fig. 6. ROC curves by fixing $L=5, S N R=-5 d B, M=6, N=8$ : (a) UNP of $0 \mathrm{~dB}$ (b) NNP of $[0,-1,1.5,-0.8,2,-1.7] d B$.
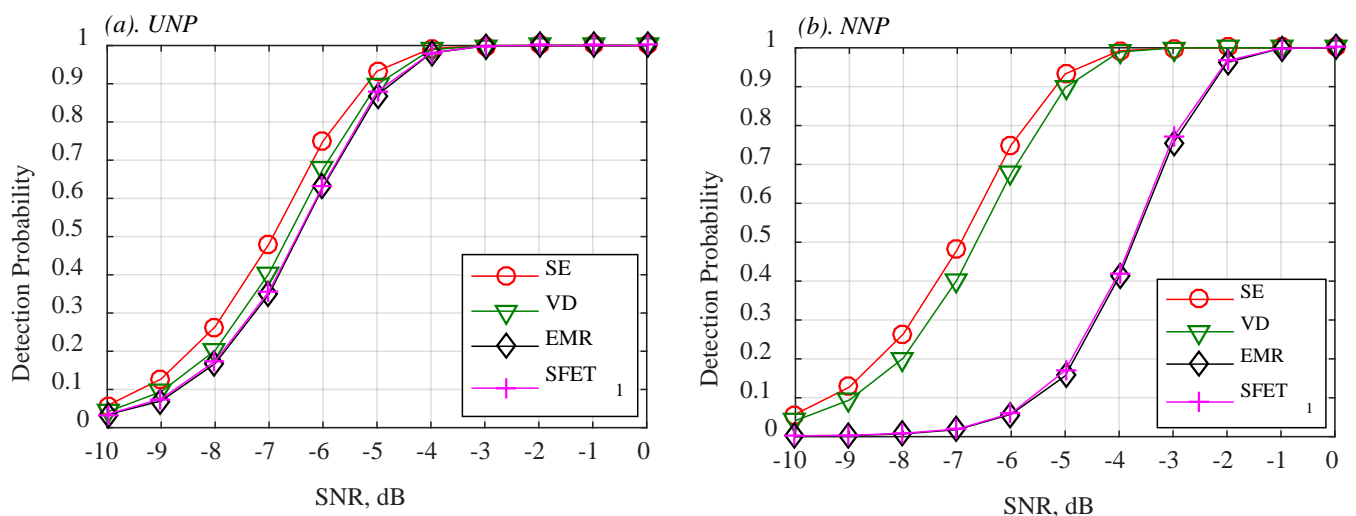

Fig. 7. Detection probabilities by fixing $L=1, M=4, N=50$ : (a) UNP of $0 \mathrm{~dB}$ (b) NNP of $[1,1.7,-0.7,-2] \mathrm{dB}$.
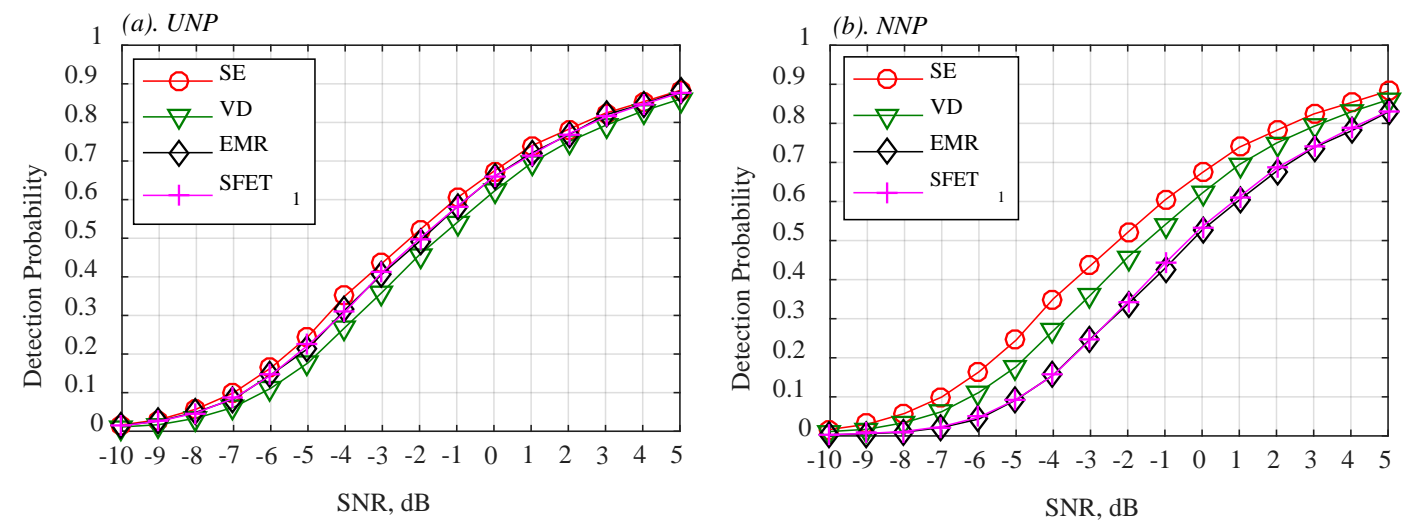

Fig. 8. Detection probabilities by fixing $L=5, M=6, N=8$ : (a) UNP of $O d B$ (b) NNP of $[0,-1,1.5$, $-0.8,2,-1.7] d B$. 


\subsection{Computational Time}

The depiction in Fig. 9 shows average computational time by fixing the number of samples at $N=50$ and varying the number of antennas. We obtained each result by averaging 50,000 independent Monte Carlo observations using a computer with 6GB RAM, Intel ${ }^{\circledR}$ Core TM i5-4690 CPU with $3.5 \mathrm{GHz}$ on a 64-bit window-7 Operating System.

Generally, under all the conditions considered, and compared with the counterpart detectors (i.e. EMR, $\mathrm{SFET}_{1}$ and VD), the proposed method has the smallest computational time since it only considers the sum of scaled-energy as given at (8). However, when the number of antennas gets very large, the computational time of the EMR and the SE are comparable and this is pointed-out using the computational complexities in Table 2: both the EMR and the SE have computational complexities of the same order.

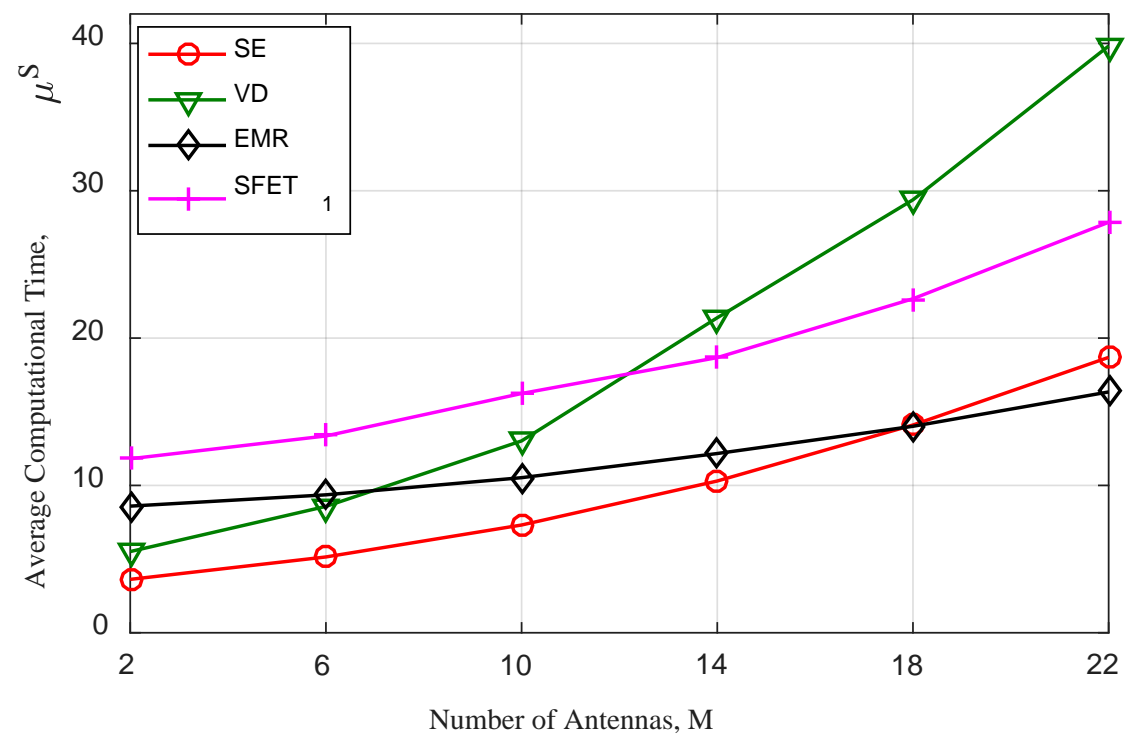

Fig. 9. Average computational time by fixing $N=50$.

\section{Conclusion}

By relying on the properties of the entries of a sample covariance matrix, we have heuristically devised a test statistic (TS) from scaled-energy. By combining theoretical results together with simulation observations, we have also approximated a decision threshold for the proposed method. Furthermore, the decision threshold does not need knowledge of noise power. The proposed method has advantages of low average computational time, high detection probability, and small Relative Error. On the top of that, by devising a new parameter which we named it 'Sensitivity of a Decision Threshold or $\beta$ ', we compared relative decision threshold deviation under the UNP case from that observed under the NNP case. And we observed that the proposed method has negligible $\beta$ value, whereas EMR and SFET $_{1}$ have large $\beta$. 


\section{References}

[1] J. Mitola, G.Q. Maguire, "Cognitive radio: making software radios more personal," IEEE Personal Communications, vol.6, no.4, pp.13-18, Aug 1999. Article (CrossRef Link).

[2] I.F. Akyildiz, W.Y. Lee, M.C. Vuran , S. Mohanty, "A survey on spectrum management in cognitive radio networks," IEEE Communications Magazine, vol.46, no.4, pp.40-48, April 2008. Article (CrossRef Link).

[3] M. Sherman, A.N. Mody, R. Martinez, C. Rodriguez, R. Reddy, "IEEE Standards Supporting Cognitive Radio and Networks, Dynamic Spectrum Access, and Coexistence," IEEE Communications Magazine, vol.46, no.7, pp.72-79, July 2008. Article (CrossRef Link).

[4] F. Khan, K. Nakagawa, "Comparative study of spectrum sensing techniques in cognitive radio networks," in Proc. of 2013 World Congress on Computer and Information Technology (WCCIT), pp.1-8, 22-24 June 2013. Article (CrossRef Link).

[5] T. Yucek, H. Arslan, "A survey of spectrum sensing algorithms for cognitive radio applications," IEEE Communications Surveys \& Tutorials, vol.11, no.1, pp.116-130, First Quarter 2009. Article (CrossRef Link).

[6] B. Wang, K.J.R Liu, "Advances in cognitive radio networks: A survey," IEEE Selected Topics in Signal Processing, vol.5, no.1, pp.5-23, Feb. 2011. Article (CrossRef Link).

[7] A.K. Kattepur, A.T. Hoang, Y.C. Liang, M.J. Er, "Data and decision fusion for distributed spectrum sensing in cognitive radio networks," Information, Communications \& Signal Processing, 2007 6th International Conference, pp.1-5, 10-13 Dec. 2007. Article (CrossRef Link).

[8] W. Zhang, Y. Guo, H. Liu, Y.J. Chen, Z. Wang, J. Mitola III, "Distributed Consensus-Based Weight Design for Cooperative Spectrum Sensing," IEEE Transactions on Parallel and Distributed Systems, vol. 26, no. 1, pp. 54-64, Jan. 1 2015. Article (CrossRef Link).

[9] E. Axell, G. Leus, E. G. Larsson, H. V. Poor, "Spectrum Sensing for Cognitive Radio : State-of-the-Art and Recent Advances," IEEE Signal Processing Magazine, vol. 29, no. 3, pp. 101-116, May 2012. Article (CrossRef Link).

[10] Y. Zeng, Y. C. Liang, "Eigenvalue-based spectrum sensing algorithms for cognitive radio," IEEE Transactions on Communications, vol. 57, no. 6, pp. 1784-1793, June 2009. Article (CrossRef Link).

[11] L. Huang, J. Fang, K. Liu, H. C. So, H. Li, "An Eigenvalue-Moment-Ratio Approach to Blind Spectrum Sensing for Cognitive Radio Under Sample-Starving Environment," IEEE Transactions on Vehicular Technology, vol. 64, no. 8, pp. 3465-3480, Aug. 2015. Article (CrossRef Link).

[12] S. Sedighi, A. Taherpour, S. Gazor, T. Khattab, "Eigenvalue-Based Multiple Antenna Spectrum Sensing: Higher Order Moments," IEEE Transactions on Wireless Communications , vol.16, no.2, pp. 1168-1184, Feb. 2017. Article (CrossRef Link).

[13] L. Huang, H.C. So, C. Qian, "Volume-based method for spectrum sensing," Digital Signal Processing, vol. 28, pp. 48-56, May 2014. Article (CrossRef Link).

[14] D. Ramirez, G. Vazquez-Vilar, R. López-Valcarce, J. Via, I. Santamaria, "Detection of Rank- P Signals in Cognitive Radio Networks With Uncalibrated Multiple Antennas," IEEE Transactions on Signal Processing, vol. 59, no. 8, pp. 3764-3774, Aug. 2011. Article (CrossRef Link).

[15] H. Wang, X. Su, J. Wang, "Cooperative spectrum sensing algorithms based on correlation matrix in cognitive radio networks," Tsinghua Science and Technology, vol. 16, no. 4, pp. 386-392, Aug. 2011. Article (CrossRef Link).

[16] Z. Li, F. Zhou, J. Si, P. Qi, L. Guan, "Feasibly efficient cooperative spectrum sensing scheme based on Cholesky decomposition of the correlation matrix," IET Communications, vol. 10, no. 9, pp. 1003-1011, June 2016. Article (CrossRef Link).

[17] R. Tandra, A. Sahai, "SNR Walls for Signal Detection," IEEE Journal of Selected Topics in Signal Processing, vol. 2, no. 1, pp. 4-17, Feb. 2008. Article (CrossRef Link).

[18] A. Abdi, M. Kaveh, "A space-time correlation model for multielement antenna systems in mobile fading channels," IEEE Journal on Selected Areas in Communications, vol. 20, no. 3, pp. 550-560, Apr 2002. Article (CrossRef Link). 
[19] M. Z. Shakir, A. Rao, M. S. Alouini, "Generalized Mean Detector for Collaborative Spectrum Sensing," IEEE Transactions on Communications, vol. 61, no. 4, pp. 1242-1253, April 2013. Article (CrossRef Link).

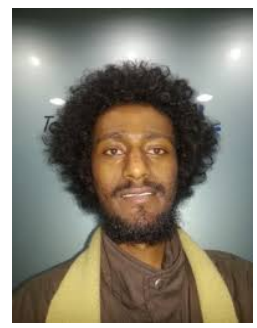

Michael Dejene Azage received his B.S. degree in Electrical and Computer Engineering from the Addis Ababa University, Ethiopia, in 2009 and he received his M.S. degree in Electrical Engineering from the Ajou University, South Korea, in 2017. Between 2014 and 2017, he worked in Multimedia Networking Lab of the Ajou University as a Researcher. His research interests include: Internet of Things (IoT), Optimization Algorithms for the Deep Learning, Robotics, and Wireless Sensor Network.

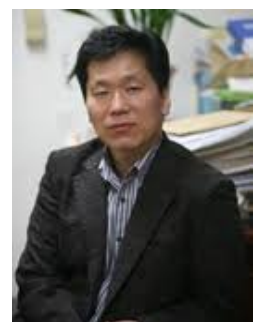

Chaewoo Lee received his B.S. degree in Control and Instrumentation Engineering from the Seoul National University in 1985; M.S. degree in Electrical and Computer Engineering from the Korea Advanced Institute of Science and Technology (KAIST) in 1988; Ph.D. in Electrical and Computer Engineering from the University of Iowa in 1995. He was with the Korea Telecom and Lucent Technologies during 1988-1999. Since March 2002, he has been with the Department of Electrical and Computer Engineering at Ajou University. His research interests lie in the realm of Multimedia Communication Systems, and High-speed Networks. 